\title{
Motion corrected LV quantification based on 3D modelling for improved functional assessment in cardiac MRI
}

\author{
Y M Liew ${ }^{1}$, R A McLaughlin 2 , B T Chan ${ }^{1}$, Y F Abdul Aziz ${ }^{3}$,

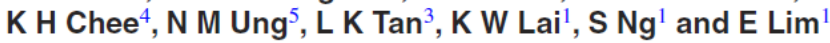

${ }^{1}$ Department of Biomedical Engineering, Faculty of Engineering, University of Malaya, 50603 Kuala Lumpur, Malaysia

${ }^{2}$ Optical+Biomedical Engineering Laboratory, School of Electrical, Electronic and Computer Engineering, The University of Western Australia, 35 Stirling Highway, Crawley, Western Australia 6009, Australia

${ }^{3}$ Department of Biomedical Imaging, University Malaya Research Imaging Centre, Faculty of Medicine, University of Malaya, 50603 Kuala Lumpur, Malaysia

${ }^{4}$ Department of Medicine, Faculty of Medicine Building, University of Malaya, 50603 Kuala Lumpur, Malaysia

${ }^{5}$ Clinical Oncology Unit, Faculty of Medicine, University of Malaya, 50603

Kuala Lumpur, Malaysia

E-mail: liewym@um.edu.my

Received 20 October 2014, revised 5 February 2015

Accepted for publication 9 February 2015

Published 13 March 2015

Abstract

Cine MRI is a clinical reference standard for the quantitative assessment of cardiac function, but reproducibility is confounded by motion artefacts. We explore the feasibility of a motion corrected 3D left ventricle (LV) quantification method, incorporating multislice image registration into the 3D model reconstruction, to improve reproducibility of 3D LV functional quantification. Multi-breath-hold short-axis and radial long-axis images were acquired from 10 patients and 10 healthy subjects. The proposed framework reduced misalignment between slices to subpixel accuracy ( 2.88 to $1.21 \mathrm{~mm}$ ), and improved interstudy reproducibility for 5 important clinical functional measures, i.e. end-diastolic volume, end-systolic volume, ejection fraction, myocardial mass and $3 \mathrm{D}$-sphericity index, as reflected in a reduction in the sample size required to detect statistically significant cardiac changes: a reduction of $21-66 \%$. Our investigation on the optimum registration parameters, including both cardiac time frames and number of long-axis (LA) slices, suggested that a single time frame is adequate for motion correction whereas integrating more LA slices can improve registration and model reconstruction accuracy for improved functional quantification especially on datasets with severe motion artefacts. 
Keywords: cine MRI, motion correction, image registration, 3D modelling, quantification

(Some figures may appear in colour only in the online journal)

\section{Introduction}

Establishing the diagnosis, treatment and prognosis of patients with cardiac disease requires accurate and reproducible quantitative measurement of left ventricular (LV) functional parameters. Research on LV remodelling, characterized by progressive LV dilatation, changes in LV shape and wall thickness or mass (Mannaerts et al 2004), has received immense interest in recent years, as it has been shown to be a good predictor for subsequent heart failure (Sebastian et al 2010). In order to accurately elucidate abnormal changes in the heart during longitudinal clinical assessments, such as those associated with LV remodelling, reliable interstudy reproducibility is of utmost importance (Grothues et al 2002). Moreover, in the context of research studies, improved accuracy and reproducibility of LV functional measurements leads to a reduction in the required sample size necessary to detect statistically significant changes in heart parameters, subsequently saving both cost and time (de Marvao et al 2014), and allows otherwise difficult studies to be undertaken (Grothues et al 2002).

The conventional LV volumetric analysis method for cardiovascular magnetic resonance (CMR) images, based on the Simpson's rule, yield significant interobserver variability due to uncertainties in defining the valvular plane and the apex (Theisen et al 2009, Carminati et al 2014) caused by movement of the valvular plane during contraction (Makela et al 2002) and partial volume effects along slice thickness (O'dell and McCulloch 2000). In view of this, subsequent studies have proposed the use of 2- and 4-chamber long-axis (LA) scans to aid in identification of the base and apex, thus reducing interstudy variability for LV functional measurements (Swingen et al 2004, Kirschbaum et al 2008, Young et al 2011). 3D cine acquisition in a single breath-hold could also be used to improve reliability of ventricular measurement by reducing the uncertainties in defining the valvular plane and apex of the heart, but at the expense of reduced resolution (i.e. spatial or temporal) or contrast-to-noise ratio (Makowski et al 2012, Jaroni et al 2013, Wech et al 2014). In contrast to conventional 2D analysis, quantification results based on 3D reconstructed models, in particular myocardial mass, have been proven to be more robust to different slice orientations during image acquisition (Frangi et al 2001, Swingen et al 2003b). Apart from conventional LV parameters, 3D models representing true LV geometry allow a more accurate quantification of shape-related parameters such as local curvature (Frangi et al 2001, Adhyapak et al 2013) and the 3D sphericity index (Mannaerts et al 2004), as well as regional variations in ventricular wall thickness and motion (Xia et al 2005, Tobon-Gomez et al 2012, de Marvao et al 2014), which could indicate myocardial abnormalities including hypertrophic cardiomyopathies or aneurysm.

Although 3D models have reduced interstudy and interobserver variability over 2D analysis methods, potential misalignment of slices may occur (Elen et al 2010), leading to deviation of the model from the true shape of the patient's heart, which is detrimental to accurate estimations of the LV parameters (Kirschbaum et al 2008). This is primarily due to inconsistent breath-hold positions and heart rhythm during acquisition (Chandler et al 2008, Elen et al 2010, Cheng-Baron et al 2014), which is exacerbated in patients with heart failure (Bellenger et al 2000a, Grothues et al 2002). To date, with the exceptions of Swingen et al (2003a) and Chandler et al (2008), studies focusing on 3D functional analysis have not considered the effect of motion artefacts in their method. While Swingen et al (2003a) attempted to minimize 
the in-plane misalignment by correcting positions of the centroids in each slice, they have not taken into account out-of-plane motions, which have been shown to be significant (Elen et al 2010, Carminati et al 2014). On the other hand, (Chandler et al 2008) demonstrated an improvement in the interstudy reproducibility for LV functional analysis by applying a sliceto-volume registration algorithm. They aligned end-diastolic, multi breath-hold SA slices in a stack with respect to each other by using a motion-free, single breath-hold volumetric scan. This single breath-hold volumetric scan, however, is not a standard scan in many centres, including ours, due to insufficient temporal resolution for the analysis of cardiac function.

Several slice-to-slice or multislice image registration approaches have therefore been suggested in the literature (Lötjönen et al 2005, Slomka et al 2007, Elen et al 2010) to reduce displacement between standard cine MRI scans. These studies either correct for in-plane translational offsets only (Slomka et al 2007), or also take into account out-of-plane translational and rotational corrections (Lötjönen et al 2005, Elen et al 2010). Despite these technological advances, most studies reported the performance of their algorithm primarily using visual comparison by 2 independent observers and the quantification of residual distances between the LA and SA epicardial contours after motion correction (Slomka et al 2007, Elen et al 2010, Carminati et al 2014). To our knowledge, interstudy reproducibility of LV functional measurements using the extracted 3D surface models has not been performed to assess the practical clinical value of these techniques.

In the present work, we propose and evaluate a novel method to perform motion corrected LV functional quantification based on a 3D surface reconstructed model. Integral to the method is a multislice-based image registration technique which extends upon earlier algorithms (Elen et al 2010) to correct for slice misalignment in multi-breath-hold cine SA-radial LA stack due to inconsistent breath-hold positions. We assess the reproducibility of LV functional parameters, including blood volume, ejection fraction, myocardial mass and 3D sphericity index, before and after motion correction, for different algorithm parameters such as number of LA slices, and demonstrate an improvement over earlier techniques utilizing only 2 LA slices. We also evaluate the robustness of the results with different numbers of cardiac time frames, to assess the trade-off between computation time and efficacy of the motion correction.

\section{Methods and materials}

\subsection{Image acquisition}

Ten healthy subjects and ten patients (10 males and 10 females, mean age: $52 \pm 10$ ) were recruited in the University of Malaya Medical Centre with written consent. All patients recruited were diagnosed with ischemic heart disease, and 6 of the cases also had myocardial infarction.

Our study protocol has received approval by University of Malaya Medical Ethics Committee (Ref: 989.75). All data were acquired on a 1.5 T MRI system (Signa HDxt 1.5 T, GE Healthcare, WI, U.S.A). For each subject, multi-breath-hold steady-state free precession SA cine image stack (FOV: $350 \times 350 \mathrm{~mm}$, image matrix: $256 \times 256$, pixel size: $1.37 \times 1.37 \mathrm{~mm}$, slice thickness: $8 \mathrm{~mm}$, gap: $0 \mathrm{~mm}$, TE/TR: $1.6 / 3.7 \mathrm{~ms}$, flip angle: $55^{\circ}$, number of slices: $10-15$, number of cardiac time frames: 20 , breath hold time: $15 \mathrm{~s}$ ) covering base to apex of the left ventricle (LV) were acquired using accelerated imaging (Array Spatial Sensitivity Encoding Technique or ASSET). A set of 6 multi-breath-hold LA cine images, containing both 2- and 4-chamber views and radially oriented around the centre of the LV chamber at uniform angular interval, were also prescribed with the same acquisition parameters by using the first SA slice at the base for planning. 
Both types of scans were acquired three times on each healthy subject and twice on each patient. All repeated scans were acquired during the same clinical scanning session, instead of during follow-up sessions. This was to avoid the effects of structural or functional change in the heart, which may be noticeable in the days after acute heart disease. Structural change could contribute to a variation in LV functions - the reproducibility of which are analysed and described in a subsequent section. Only two repeated scans were performed on patients in view of high patient load at the clinics and long acquisition time for each patient as other standard clinical scans (e.g. LGE and stress/rest perfusion scans) were also acquired for clinical diagnosis. Overall, there were a total of 50 sets of data for registration and assessment of reproducibility.

\subsection{Image registration algorithm}

The aim of this study is to correct for misaligned SA and LA slices simultaneously by performing a multislice rigid registration. This registration is fully automated and is based on the assumption that similarity of the intensity profiles along the intersecting lines (Elen et al 2010) of both types of scans is optimized when they are in correct alignment.

All SA and LA slices were initially transformed into the reference coordinate system of the heart based on the position information retrieved from the DICOM file header. As the cine sequence of slices at a specific location was acquired within the same breath-hold, we have assumed there is negligible misalignment within each co-located cine sequence. However, motion of the patient between breath-holds leads to the erroneous positioning of both the LA and SA slices in this reference coordinate system. The registration framework here seeks to rectify such slice displacements.

Our registration framework consists of a 3D rigid transformation which corrects for 6 degree-of-freedom motion, including translational motions $t_{x}, t_{y}$ and $t_{z}$ along $x, y$ and $z$ axes and rotational motions $\alpha, \beta$ and $\gamma$ about these axes. The transformation matrix $T_{\text {rigid }}$ which transforms a voxel $x$ in the image can be represented as follow (Chandler et al 2008):

$$
T_{\text {rigid }}(x)=R x+t
$$

where the translation $t$ is given by:

$$
t=\left(t_{x}, t_{y}, t_{z}\right)^{T}
$$

and the rotation matrix $R$ is given by:

$$
R=\left(\begin{array}{ccc}
\cos \beta \cdot \cos \gamma & \cos \alpha \cdot \sin \gamma+\sin \alpha \cdot \sin \beta \cdot \cos \gamma & \sin \alpha \cdot \sin \gamma-\cos \alpha \cdot \sin \beta \cdot \cos \gamma \\
-\cos \beta \cdot \sin \gamma & \cos \alpha \cdot \cos \gamma-\sin \alpha \cdot \sin \beta \cdot \sin \gamma & \sin \alpha \cdot \cos \gamma+\cos \alpha \cdot \sin \beta \cdot \sin \gamma \\
\sin \beta & -\sin \alpha \cdot \cos \beta & \cos \alpha \cdot \cos \beta
\end{array}\right)
$$

During registration, the intersection point of all LA slices with each SA slice was used as the centre of rotation for that SA slice. By contrast, the intersection point of the middle SA slice with all LA slices was used as the centre of rotation for all LA slices.

Since the intensity range of different slices could differ due to the inhomogeneity in the magnetic field, the same anatomical structure may appear with different intensity in different slices. The similarity measures for all intersection lines between LA and SA slices, therefore, are evaluated using the normalized cross-correlation which is a registration similarity measure that accounts for linear intensity fluctuation across an image sequence (Makela et al 2002).

For optimal cardiac motion correction, alignment of SA and LA slices should be based on relevant cardiac structures instead of the surrounding tissues such as liver, lung and thoracic 
cage as the appearance of these tissues varies with the level of expiration. A region of interest (ROI) mask was produced to exclude such structures from the similarity calculation. In contrast to earlier algorithms (Elen et al 2010), where the use of a standard ROI contour may require some empirical modifications between datasets, we have used a fully-automated algorithm to define ROI. This ROI encompasses both LV and RV with the aim to reduce ambiguous optimal registration due to subtle change in LV along the long-axis, which is particularly evident at the base and midventricular sections. In our work, the ROI mask for each slice was computed by first calculating the variance image along the time axis (i.e. calculating variance in intensity at each location within a slice across all time frames). This provided an image in which moving objects, predominantly the heart region, produced high image intensity variance values over time, and the surrounding stationary tissue regions had negligible variance.

The variance image was thresholded and morphologically processed to produce a binary mask. The foreground region of the mask was subsequently modelled with an ellipse to delineate a region of interest encompassing the heart. All pixels within this region were assigned a weight of 1 . The mask was then spatially smoothed such that the smoothed mask contained values between 0 and 1 . This is accomplished by morphologically dilating the ellipse mask. The region outside this larger ellipse was assigned a value of 0 whereas the weights in the remaining unassigned region were linearly interpolated between 0 and 1 . These weightings were used to modify the impact of each pixel on the registration algorithm. Using such a mask, where weights vary smoothly from 0 to 1 , robustness of the registration algorithm was improved (Elen et al 2010). A single empirically chosen dilation factor was used for all slices to ensure that all cardiac structures over the cardiac cycle were within the two ellipses. Figure 1 illustrates the variance images and ROI masks computed for both SA and LA slices. The ROI mask defined for each slice covers the entire LV as well as both in-flow and out-flow tracts. This mask was kept the same for all cardiac time frames and for all experiments.

During the calculation of the similarity function for the relative positioning of each pair of intersecting slices, an ROI weighting mask as described above was utilized for one slice, while in the other slice a uniform mask with all weights set to 1 was utilized. The normalized cross-correlation was computed over pixels at the intersection of the two slices weighted by the product of the masks, which is given by the following equation (Elen et al 2010):

$\mathrm{NCC}=\frac{\sum_{k=1}^{K} W_{n \cap m}(k) \cdot\left(S_{n \cap m}^{n}(k)-\mu S_{n \cap m}^{n}\right) \cdot\left(S_{n \cap m}^{m}(k)-\mu S_{n \cap m}^{m}\right)}{\sqrt{\sum_{k=1}^{K} W_{n n m}(k) \cdot\left(S_{n n m}^{n}(k)-\mu S_{n \cap m}^{n}\right)^{2}} \cdot \sqrt{\sum_{k=1}^{K} W_{n n m}(k) \cdot\left(S_{n \cap m}^{m}(k)-\mu S_{n \cap m}^{m}\right)^{2}}}$

where $S_{n \cap m}^{n}$ and $S_{n n m}^{m}$ are the intensity profiles of slice $n$ and $m$, respectively, along their intersection. $K$ is the number of samples in the profile. $\mu S_{n n m}^{n}$ and $\mu S_{n n m}^{n}$ are the means of $S_{n n m}^{n}$ and $S_{n n m}^{m}$ , respectively. $W_{n \cap m}$ is the weight obtained as the product of the intensities of the masks along the intersection line of the slices $n$ and $m$.

The similarity function was optimized through a minimization process, where the cost function to be minimized was cost $=1-\mathrm{NCC}$, where NCC is the normalized cross correlation. This cost is a measure of the dissimilarity of the intensities along the intersection line. The total cost function, to be minimized with respect to the $3 \mathrm{D}$ translational and rotational parameters, was given by the sum of the cost functions from all pair of intersecting slices (including both short-axis and long-axis slices) over all cardiac time frames. The minimization process was performed using a Quasi-Newton optimizer (Flannery et al 1992), in which the optimal step size for minimization was calculated using a line search method. The finite-differencing 


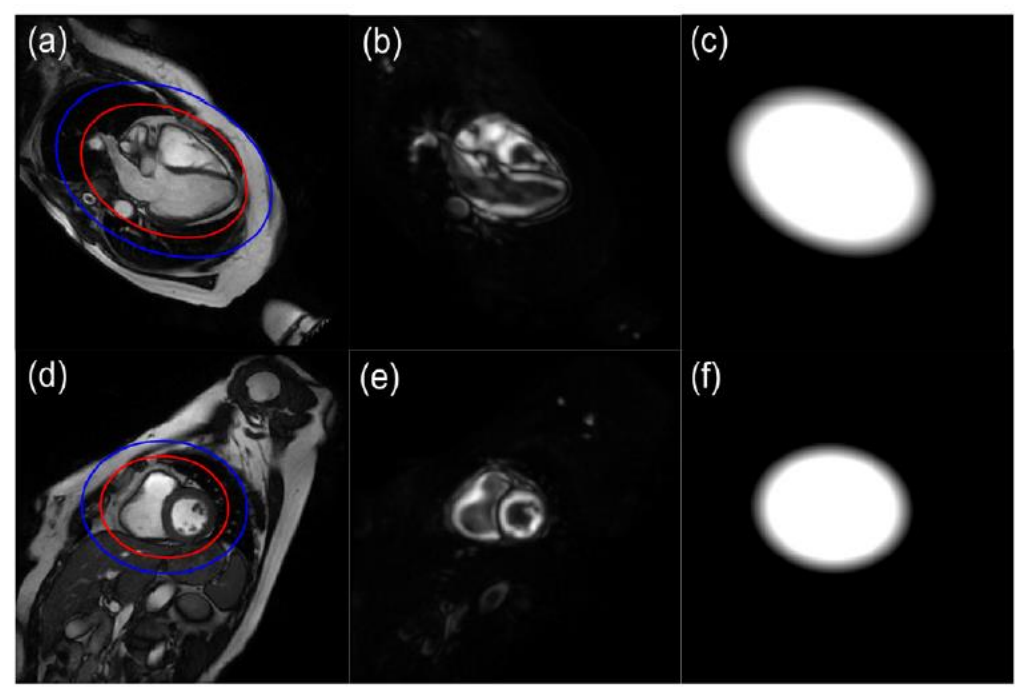

Figure 1. ROI definitions for LA scan (top row) and SA scan (bottom row). The variance image and ROI mask of the LA scan in $(a)$ are shown in $(b)$ and $(c)$, respectively. For SA scan in $(d)$, the corresponding variance image and ROI mask are shown in $(e)$ and $(f)$, respectively. The region inside the red ellipse is assigned a weight of 1 . The region of the image outside the blue ellipse is given weight 0 and weights in the intervening region are interpolated between 1 and 0 . (See text for details)

method was used to calculate and update the gradient and Hessian matrices during each iteration process. All slices, including both SA and LA slices, were simultaneously registered relative to a static reference LA slice (4-chamber view). Transformation parameters for each slice are independent and so were estimated simultaneously. The registration framework was implemented in MATLAB (vR2012a, Mathworks, Natick, MA) on an Intel(R) Core(TM) i5-3570 CPU @3.40 GHz computer.

The number of cardiac time frames (or cardiac phases) and number of LA slices used for registration could impact both registration accuracy and computation time. To investigate the effect of the former, various cardiac time frames (i.e. 1, 5, 10, 15 and 20 frames) were tested during registration as guided by all LA slices (i.e. 6 slices) available in this study. Specifically, the image used was from the first time frame (i.e. diastolic phase whereby the LV geometrical changes is minimal) when only one time frame was investigated. When multiple time frames were used, these were spaced equidistant in time over the R-R peaks. The effect of the latter was investigated by varying the number of LA slices for registration (i.e. 2, 3, 4, 5 and 6 slices) while fixing the time frame to 1 (i.e. the first time frame). In each case, the slices were selected to be equally spaced radially. Computation times for registrations were also recorded.

\subsection{Assessment of registration accuracy}

The proposed registration framework was validated using all 50 data sets from both healthy subjects and patients. The accuracy of the registration algorithm was quantified as the average 
shortest residual displacement error between LV epicardial contours, which were manually segmented and verified by a radiologist from both SA and LA slices using editable userplaced guide-points in Segment (free version 1.9 R3136, Medviso, AB) (Heiberg et al 2010, Young et al 2011). The error measure was calculated for both end-diastolic and end-systolic phases as these cardiac phases were used most often for functional analysis during clinical diagnosis. Note that these contours were used only for validation purposes and were not used for registration. The average residual displacement error before and after registration from all subjects was reported as the registration accuracy. The change in the registration accuracy using different numbers of LA slices were tested for statistical significance using Friedman's test, followed by post-hoc analysis with Wilcoxon signed-rank test with a Bonferroni correction. Significant level was set at 0.05 .

\subsection{Reproducibility study}

Epicardial and endocardial LV contours were manually delineated from all slices and reconstructed into a 3D surface model. During delineation of the endocardial contour, papillary muscles and trabeculae were included as part of the myocardial wall in situations where it was difficult to distinguish them from the wall. By contrast, if these structures could be seen clearly and were enclosed within the blood pool, they were assigned to the LV cavity (Gandy et al 2008). To reconstruct the model, all contours were initially registered in the reference DICOM coordinate system. The model was then generated by fitting closed and open cubic B-spline curves along the circumferential and longitudinal direction, respectively, based on the algorithm proposed by de Boor (De Boor 1972, Hoschek et al 1993). Base and apex regions beyond SA slices were reconstructed based on LA contours, while other regions were based on SA contours.

The end-diastolic $\left(V_{\mathrm{ed}}\right)$, end-systolic $\left(V_{\mathrm{es}}\right)$ and myocardial tissue volume were then computed using a surface integration method based on the Divergence Theorem of Gauss (Kreyszig 2007), which relates the volume of a closed surface to the surface integral. Myocardial mass (mass) was calculated by multiplication of myocardial tissue volume by the specific density of myocardium (i.e. $1.05 \mathrm{~g} \mathrm{~cm}^{-3}$ ) (Semelka et al 1990, Bogaert et al 1995), whereas the ejection fraction $(\mathrm{EF})$ of the $\mathrm{LV}$ was calculated using the following formula:

$$
\mathrm{EF}=\frac{V_{\mathrm{ed}}-V_{\mathrm{es}}}{V_{\mathrm{ed}}}
$$

To estimate the global LV shape, the 3D sphericity index (SI) was calculated from the LV cavity at end-diastole (Mannaerts et al 2004). SI was obtained as the $V_{\text {ed }}$ divided by the volume of a sphere in $\mathrm{mL}$, whereby the diameter of the sphere was defined as the major long-axis of the cavity. This major long-axis can be derived from the 3D reconstructed surface model as the longest distance between the center of mass of the mitral annulus and the endocardial apex.

For each subject, the signed difference in the $V_{\mathrm{ed}}, V_{\mathrm{es}}, \mathrm{EF}$, mass and SI between all repeated cine scans was computed both before and after registration. These difference values were then averaged over the group of healthy subjects and patients separately to give the mean difference for each group. The standard deviation (SD) of the difference values, which is taken as the interstudy reproducibility of the functional metric, was also calculated for each group (Chandler et al 2008).

Statistical significant differences of the functional measurements before and after motion correction were analysed using the Statistical Package for Social Sciences (SPSS v21.0, SPSS Inc., USA). Data were tested for normality beforehand using Shapiro-Wilk test. As the data do not exhibit normal distribution, non-parametric Wilcoxon-Signed Rank test were therefore 


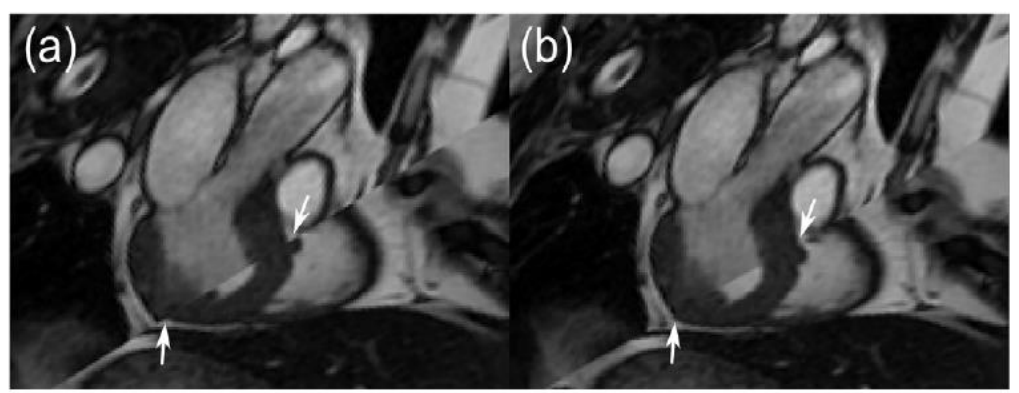

Figure 2. Example of intersecting LA and SA slices before $(a)$ and after registration (b) using 6-degrees-of-freedom registration algorithm. Arrows indicate where the registration algorithm decreased slice misalignment.

performed. Similarly, such significant tests were performed before and after motion correction on the difference values for repeated scans after these values were squared to remove the sign (Grothues et al 2002).

For the calculation of sample size required to detect a clinical difference in LV functional metrics before and after registration, we have adopted the method of Chandler et al (Chandler et al 2008). The sample size needed to show a clinical difference $(\delta)$ with a power $(P)$ of $90 \%$ and an error $(\alpha)$ of 0.05 is given by equation (6):

$$
n=\frac{2 \cdot f(\alpha, P) \cdot \sigma^{2}}{\delta^{2}}
$$

where $n$ is the sample size, $P$ is the study power required to detect a significant changes, $\alpha$ is the significant level, $f$ is the factor for different values of $\alpha$ and $P($ e.g. $f(0.05,0.90)=10.5), \sigma$ is the interstudy reproducibility (i.e. SD of the difference values), and $\delta$ is the desired difference to be detected. To be consistent with the previous studies (Semelka et al 1990, Bogaert et al 1995, Bellenger et al 2000a, Chandler et al 2008), $\delta$ was fixed at $10 \mathrm{ml}$ for both $V_{\text {ed }}$ and $V_{\mathrm{es}}, 3 \%$ absolute change for $\mathrm{EF}, 10 \mathrm{~g}$ for mass and $10 \%$ for SI.

\section{Results}

\subsection{Registration accuracy}

Figure 2 illustrates two intersecting LA and SA slices before and after registration. While there is a clear misalignment between these orthogonal slices before registration, the displacements between SA and LA slices were reduced with the use of 6 degree-of-freedom registration algorithm. The recovered $3 \mathrm{D}$ translation and rotation using the multislice registration approach for our 50 data sets was found to range from $-15 \mathrm{~mm}$ to $13 \mathrm{~mm}$ and $-7^{\circ}$ to $6^{\circ}$, respectively.

Figure 3 shows the LV surface models of a healthy subject and a patient before and after registration at the end-diastolic phase by using 6 radial LA slices and a parallel SA stack for registration and model reconstruction. It can be observed that radial LA slices provide supplementary information necessary to reconstruct the base and apex of the LV. The presence of motion artefacts has apparently misaligned both SA and LA slices as evidenced by the offsets between the LV contours segmented from both types of scans (i.e. cyan and blue dotted lines). 


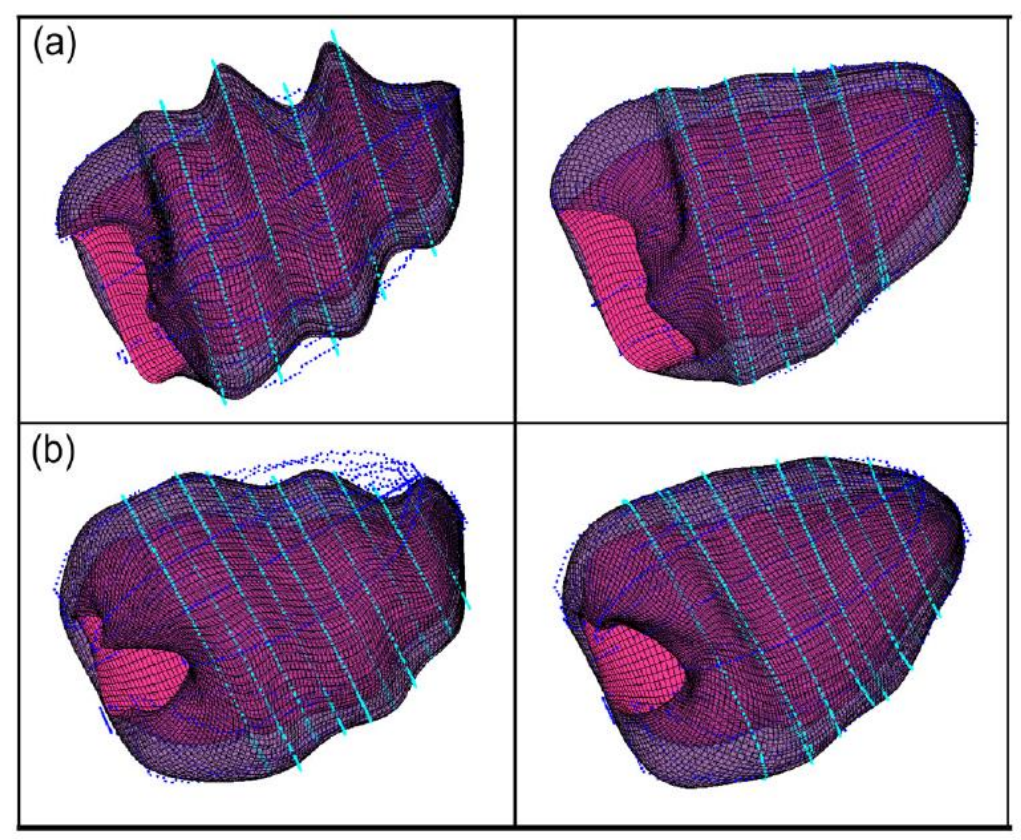

Figure 3. Reconstructed 3D surface geometries of LV of $(a)$ normal subject and $(b)$ patient before and after motion correction. The cyan and blue dotted lines indicate the LV contours segmented from SA and LA slices, respectively. Note the large displacements between LV contours from both type of scans before registration (left) and the corresponding reduction in displacements after registration (right).

Therefore, subsequent model reconstruction based on these contours has led to the deformation of the model (figure 3, left column) and the deviation of functional analysis from the true value. Simultaneous registration of all slices using our registration framework has shown to reduce such misalignments and improve the fidelity of the model to the true LV geometry (figure 3, right column).

Our preliminary study indicated that the total amount of motion artefacts affecting both healthy subjects and patients was comparable. Very similar registration accuracies were also obtained for both groups and therefore their results were pooled together. The box-whisker plot in Figure 4 shows the average residual displacement error and computational time plotted against different numbers of cardiac time frames for all subjects. The median displacement error before registration was computed to be $2.83 \mathrm{~mm}$ with a maximum error of $\sim 40 \mathrm{~mm}$. Registration using the proposed framework has reduced the median residual displacement error significantly $(p<0.05)$ to $1.22 \mathrm{~mm}$ and the maximum error to $\sim 28 \mathrm{~mm}$. This remaining median error was found to be similar $(p>0.05)$ using more than one cardiac time frame and was comparable in magnitude to the MRI spatial resolution, with a single pixel being $1.37 \mathrm{~mm}$. The computational time recorded for registration using all 20 cardiac time frames was $\sim 4.7 \mathrm{~h}$, which is approximately $4-5$ times longer than the time required when only a single time frame was used (i.e. $\sim 1$ h) in a non-optimized MATLAB implementation. 


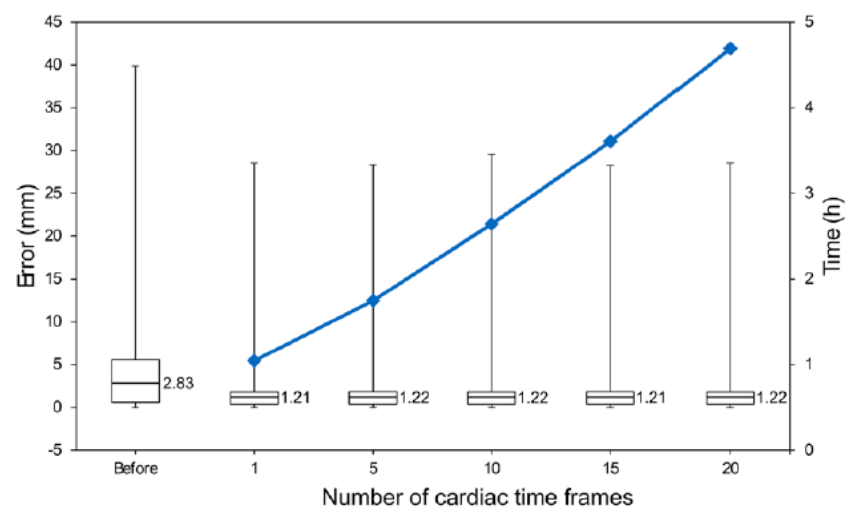

Figure 4. Effect of the number of cardiac time frames on registration accuracy and computation time. Box-whisker plot indicates the median, interquartile range, minimum and maximum residual displacement errors between LA and SA contours before and after registration using a different number of cardiac time frames on 50 data sets. The number of LA slices was fixed at 6. The median value is reported next to each box-plot. The line plot in blue indicates the computational time, in hours, when different numbers of time frames are used in the registration process.

In figure 5, the number of LA slices was shown to affect the registration accuracy, with more LA slices yielding improved accuracy. The median residual displacement error was reduced from $2.88 \mathrm{~mm}$ to $1.55 \mathrm{~mm}$ when only $2 \mathrm{LA}$ slices (i.e. 2- and 4-chamber views) were used. When $6 \mathrm{LA}$ slices were used, a minimum error of $1.22 \mathrm{~mm}$ was obtained. The improvement in the registration accuracy was found to be significant $(p<0.05)$ when comparing before and after registration, as well as between registrations using different number of LA slices, except between $2 \mathrm{LA}$ and $3 \mathrm{LA}$ slices $(p=0.48)$. The improvement in registration accuracy from 2 to 6 LA slices, however, is at the cost of increased computation time. Using the current registration framework without speed optimization, registration using 2 LA slices requires a computation time of $\sim 18 \mathrm{~min}$, whereas registration using $6 \mathrm{LA}$ slices requires $\sim 1 \mathrm{~h}$. Although the maximum error remains high after registration (figures 4 and 5), this was noted to be indicative of approximately $10 \%$ of the data sets, where blurring of the SA and LA slices led to a poor registration result.

\subsection{Reproducibility study}

Table 1 shows the mean, standard deviation and the range of the $V_{\mathrm{ed}}, V_{\mathrm{es}}$, EF and mass before and after motion correction for the 2 study groups. These functional measures were computed from the 3D models generated using LV contours extracted from both LA and SA slices. Only measures from 3D models produced from 2 and 6 LA slices are shown, since the former was the most commonly used number of LA slices in previous studies (Swingen et al 2004, Slomka et al 2007, Elen et al 2010), whereas the latter was chosen because it yielded the smallest residual displacement error in our current study. In general, at p level of $0.1, V_{\text {ed }}$, $V_{\mathrm{es}}$, mass and SI were found to be significantly changed after motion correction in healthy subjects. For patient, $V_{\text {ed }}, V_{\text {es }}$, mass and SI were significantly changed when 2 LA slices were

\section{Link to Full-Text Articles :}

http://www.ncbi.nlm.nih.gov/pubmed/25768708

http://iopscience.iop.org/0031-9155/60/7/2715/pdf/0031-915560 7 2715.pdf 